\title{
LOS GRUPOS FINANCIEROS EN EL ECUADOR - 25 AÑOS DESPUÉS
}

\author{
FINANCIAL GROUPS IN \\ ECUADOR - 25 YEARS LATER
}

LUIS FIERRO CARRIÓN ${ }^{1}$

Recibido: 12 de abril

Aceptado: 1 de mayo

$\overline{1}$ Economista, M.A., M.Sc.Econ., Ph.D. (c). Universidad Andina Simón Bolívar. 



\section{LOS GRUPOS FINANCIEROS EN EL ECUADOR - 25 AÑOS DESPUÉS ${ }^{1}$}

FINANCIAL GROUPS IN ECUADOR - 25 YEARS LATER

Luis Fierro Carrión

Palabras Clave: Grupos Económicos, Grupos Financieros, Concentración y Centralización del Capital, Capitalismo Monopolista de Estado, Poder de Mercado

Keywords: Economic Groups, Financial Groups, Concentration and Centralization of Capital, State Monopoly Capitalism, Market Power

\section{RESUMEN}

Hace 25 años, el CEDEP publicó Guillermo Navarro (1976), así como los mi libro Los Grupos Financieros en el Ecuador. El libro, con 665 páginas, era una versión resumida y editada de mi Tesis de Grado como Economista de la Pontificia Universidad Católica del Ecuador (PUCE), presentada en agosto de 1989.

El estudio construía sobre los trabajos previos de David Hanson (1971) y avances efectuados por José Moncada, Patricio Ruiz e investigadores del Instituto de Investigaciones Económicas de la Universidad Central. También fue vital el aporte del proyecto de investigación "La Concentración y Centralización del Capital en el Ecuador", auspiciado por el CONUEP, en el cual participé junto con

\footnotetext{
1 Publicado en MPRA, 2017, Paper No. 81306; recuperado de: https://mpra.ub.uni-muenchen.de/81306/
} 
Diego Borja, Diego Mancheno, Martha Moncada y Pedro Páez, entre otros.

La base teórica era que en el Ecuador se había alcanzado la fase del "capitalismo monopolista de Estado", concepto de Lenin que se había desarrollado en Francia y en México. La intervención del Estado en el proceso de acumulación se vuelve permanente e irreversible, para garantizar la reproducción del capital. Este concepto es consistente también con el modelo de "capitalismo de Estado" impulsado por China y otros países BRICS (Brasil en nuestro continente).

En la última década de gobierno de Alianza PAIS, si bien se ha utilizado retórica como la del "socialismo del siglo XXI" y "revolución ciudadana", en la práctica el proceso de fortalecimiento del capitalismo monopolista de Estado, y de los grupos financieros, se ha acelerado. Durante los años de auge del precio internacional del petróleo la participación del Estado en la economía aumentó, y la mayoría de los grupos económicos se han visto fortalecidos, con excepción de un par de grupos (Isaías, Álvaro Noboa) que se han visto sujetos de expropiaciones y juicios. Pero otros grupos han tenido un apogeo, destacándose entre ellos los grupos Wright (Corporación Favorita), Eljuri, NOBIS (Isabel Noboa), Hidalgo, y otros grupos vinculados al comercio y la construcción.

Se constituyó la Superintendencia de Control de Poder de Mercado, junto a la aprobación de su respectiva Ley, la cual ha impuesto algunas multas a empresas y requerido que la etiquetación de ciertos productos se modifique. Pero no ha determinado, hasta la fecha, medidas que afecten en su esencia a los grupos económicos, los oligopolios o su poder de mercado.

Un cambio legal importante fue la desvinculación de las instituciones financieras de otro tipo de compañías, al prohibirse que los accionistas de entidades financieras pudiesen a la vez invertir en otros tipos de entidades, por mandato constitucional. No obstante, se mantienen lazos evidentes entre ciertos grupos económicos y determinadas instituciones financieras (por ejemplo, entre el Banco del Austro y el Grupo Eljuri).

Otro cambio importante, especialmente para la investigación económica, fue que el Servicio de Rentas Internas (SRI) comenzó a compilar y publicar información sobre los grupos económicos. En mi caso, y me imagino que el de otros investigadores en décadas pasadas, se requería una labor de hormiga, recortando balances publicados en la prensa, folletos publicados por los grupos financieros, información sobre miembros de directorios, y hasta direcciones de correo postal, para poder investigar; ahora, basta con ir al sitio de Internet del SRI y de la Superintenden- 
cia de Bancos para obtener información oficial. Se ha avanzado mucho en la investigación sobre el tema, con varias

Tesis de Grado y libros publicados.

\section{ABSTRACT}

25 years ago, CEDEP published my book, The Financial Groups in Ecuador. The book, 665 pages long, was a condensed and edited version of my thesis as an Economist from the Pontifical Catholic University of Ecuador (PUCE), presented in August 1989.

The study built on previous works by David Hanson (1971) and Guillermo Navarro (1976), as well as progress made by José Moncada, Patricio Ruiz and researchers of the Institute of Economic Research of the Central University. The contribution of the research project "The concentration and centralization of capital in Ecuador," sponsored by the CONUEP, was also vital; Diego Borja, Diego Mancheno, Martha Moncada, Pedro Páez, and I, among others, participated in this project.

The theoretical basis was that Ecuador had reached the stage of "state monopoly capitalism" a concept of Lenin that had been further developed in France and Mexico. State intervention in the accumulation process becomes permanent and irreversible, to ensure capital reproduction. Interestingly, this concept is also consistent with the model of "sta- te capitalism" driven by China and other BRICS (Brazil in our continent).

In the last decade of government of Alianza PAIS, despite rhetoric such as the "21st century socialism" and "citizens' revolution", in practice the process of strengthening of state monopoly capitalism, and financial groups, has accelerated. Although during the boom years of international oil prices state involvement in the economy increased, most economic groups have also strengthened, with the exception of a couple of groups (Isaias, Alvaro Noboa) that were subject of expropriations and lawsuits. But other groups have had a heyday, prominent among them the Wright (Corporación Favorita), Eljuri, NOBIS (Isabel Noboa), Hidalgo and other groups linked to commerce and construction.

While the Superintendence of Control of Market Power was established, together with the approval of the respective Law, it has imposed some fines to companies and required the labeling of certain products be changed; but it has not been adopted to date, measures to break up economic groups, oligopolies or abuse of market power. 
An important legal change was the separation of financial institutions from other companies, by prohibiting the shareholders of financial institutions to invest in other types of entities, by constitutional mandate. However, clear links between certain economic groups and certain financial institutions continue (for example, between Banco del Austro and the Eljuri Group).

Another important change, especially for economic research, was that the Internal Revenue Service (SRI) began compiling and publishing information on economic groups. In my case, and I imagine that of other researchers in past decades, the research process was a grinding work, cutting balances in the press, pamphlets published by financial groups, information about board members, and even company addresses; now, you can obtain official information on the web sites of the SRI and the Superintendence of Banks. There has also been progress in research on the subject, with various theses and books published.

Ficha del Autor: El autor es un especialista en el desarrollo económico, social y ambiental de América Latina y el
Caribe. Ha sido Asesor en Temas de Financiamiento para enfrentar el Cambio Climático para la Asociación Independiente de Latinoamérica y el Caribe (AILAC). Fue funcionario durante 17 años del Banco Interamericano de Desarrollo (BID). Se desempeñó como Coordinador de Movilización de Recursos en la Oficina en Europa del BID. Anteriormente fue Coordinador Principal de Programas en el Departamento de Desarrollo Sostenible del BID, donde participó en la elaboración de estrategias sobre crecimiento sostenible, reducción de la pobreza, medio ambiente y competitividad, entre otros temas. Se graduó como Economista por la Pontificia Universidad Católica del Ecuador, y obtuvo títulos de postgrado en Desarrollo Económico, Economía Internacional y Economía Ambiental en la Universidad de Oregón (M.A.) y la Universidad de Texas en Austin (M.Sc.Econ., Ph.D. (c)). Ha escrito, contribuido, editado y traducido numerosos libros, ensayos y artículos, sobre temas del desarrollo económico, social, ambiental e institucional. En 1991, publicó el libro "Los Grupos Financieros en el Ecuador" (CEDEP), y ha publicado otros libros y artículos sobre la concentración y centralización del capital en el país. 


\section{INTRODUCCIÓN}

Hace 25 años, el Centro de Educación Popular (CEDEP) publicó mi libro Los Grupos Financieros en el Ecuador. El libro, de 665 páginas, es una versión resumida y editada de mi Tesis de Grado como Economista de la Pontificia Universidad Católica del Ecuador (PUCE), presentada en agosto de 1989.

Unos años antes, en noviembre 1986, el Centro de Estudios y Difusión Social (CEDIS, en ese entonces vinculado al CEDEP) había publicado otra obra mía, Los Grupos Monopólicos en el Ecuador, concebido como un ensayo de difusión popular, pero que ya presentaba una radiografía de los grupos económicos del país hacia 1984, su interrelación con el Estado y con el capital transnacional (lo publiqué anónimamente, para preservar el acceso a fuentes de información, y también para no poner en riesgo a las personas que me habían provisto de información).

Sin lugar a duda, los dos trabajos construían sobre la base del estudio de Guillermo Navarro, La Concentración de Capitales en el Ecuador (Navarro, 1976), que realizó una primera caracterización de los principales grupos económicos del país. David Hanson (1971) también había identificado diez "imperios económicos", principalmente vinculados a la agro- exportación de la Costa.
Otros avances importantes tuvieron lugar en el Instituto de Investigaciones Económicas (IIE) de la Universidad Central, bajo el liderazgo de José Moncada (quien fuera también Decano de la Facultad de Economía y posteriormente Rector de la Universidad Central), quien publicó varios estudios sobre la burguesía monopólica. Otros investigadores del IIE incluían a Marco Tafur, Isaías Campaña, Patricio Ruiz, Lucas Pacheco, entre otros.

El IIE también auspició el proyecto de investigación del CONUEP, "La Concentración y Centralización del Capital en el Ecuador", en el cual participé junto con Diego Borja, Diego Mancheno, Martha Moncada, Pedro Páez, entre otros. Diego Mancheno había escrito unos años antes su Tesis de Grado, De la Concentración y Centralización del Capital al Capitalismo Monopolista de Estado: un Acercamiento al Caso Ecuatoriano (1986)

El concepto de "capitalismo monopolista de Estado" (CME), inicialmente propuesto por Lenin, luego fue desarrollado en países como Francia y en México (por autores como Paul Bocarra y Alonso Aguilar). Se sustentaba en afirmar que la intervención del Estado en el proceso de acumulación se había vuelto permanente e irreversible, para garantizar la reproducción del capital. El CME surge como respuesta a la creciente contradicción entre 
la socialización de las fuerzas productivas, y el régimen privado de apropiación.

Curiosamente, este concepto es consistente también con el modelo de "capitalismo de Estado" impulsado por China y otros países BRICS (Brasil en nuestro continente). En estos países, el Estado tiene una presencia importante en los sectores productivos y financieros, a través de empresas de propiedad estatal, así como bancos públicos.

En esta ponencia, al igual que en mi libro, el término "grupo financiero" se refiere a un conglomerado de empresas industriales, comerciales, financieras, de transporte, agropecuarias, etc. que se han constituido en los centros fundamentales del poder económico y político (Del Llano, 1978, p. 140). Tras la separación de las instituciones financieras de las empresas de otros sectores, se podría hablar de "grupos económicos", por una parte, y "grupos financieros", por otra, como lo hacen el SRI y la SB. No obstante, continuaré utilizando los dos términos de manera intercambiable (con la salvedad de que algunos grupos financieros concentran sus actividades exclusivamente en el sector financiero).

En la última década de gobierno de Alianza PAIS, si bien se ha utilizado retórica como la del "socialismo del siglo $X X I$ ", en la práctica el proceso de fortalecimiento del capitalismo monopolista de Estado (CME), y de los grupos financieros, se ha acelerado. Si bien durante los años de auge del precio internacional del petróleo la participación del Estado en la economía aumentó, la mayoría de los grupos económicos también se han visto fortalecidos, con excepción de un par de grupos (Isaías, Álvaro Noboa) que han sido sujetos de incautaciones, embargos y juicios, o han quebrado por otros motivos (Ortega, Tosi). Pero otros grupos han tenido un apogeo, destacándose entre ellos los grupos Wright (Corporación Favorita), El Rosado, Eljuri, NOBIS (Isabel Noboa), Hidalgo \& Hidalgo, y otros grupos vinculados a la importación, el mercado interno y la construcción.

En los últimos años se ha avanzado mucho en la investigación sobre el tema, con varias Tesis de Grado y libros publicados, entre ellos el de Carlos Pástor Pazmiño (2015), que citaré en mi ponencia. Otros trabajos que cabe mencionar son las actualizaciones sobre el tema que ha publicado Napoleón Saltos; un nuevo libro de Guillermo Navarro sobre los "Poderes Fácticos" (2006); Totoy y Vizuete (2012); Rosero Delgado (2013); Villalta (2014); los informes periódicos del SRI y la Superintendencia de Bancos; y artículos en la Revista "Gestión", Revista "Ekos", los diarios "El Universo", "El Comercio", y“El Telégrafo", entre otros. 


\section{PRINCIPALES GRUPOS FINANCIEROS}

Los primeros grupos económicos familiares en el Ecuador se constituyeron en torno a la agro- exportación, incluyendo los "Gran Cacao" de finales del siglo XIX e inicios del XX, y los grandes exportadores de banano, café, cacao y otros productos primarios a mediados del siglo XX. En la Sierra, los grandes terratenientes gradualmente incursionaron en el comercio, la banca, y posteriormente la industria.

Hanson (1971) ya hablaba de diez "imperios económicos", destacando en particular el poder de los bancos La Previsora y del Descuento, que en la década de 1960 llegaron a detentar el 72\% de los depósitos. Los 10 "imperios" eran: Noboa Naranjo (13 empresas), Estrada Ycaza (26 empresas), Vallarino (9), Ycaza Cornejo (20), Maspons (6), Pérez Valdez (48), Ecuadorian Corporation (11), Maulme Gómez (6), Cañarte Barbero (16) y Orces Mendoza (8). Estos diez grupos poseían 55 grandes industrias, 22 grandes firmas comerciales, y las 11 principales entidades financieras. Hanson también se refiere a la "oposición libanesa", con participación creciente en el comercio, la industria textil y las finanzas.

Navarro (1976) identifica dos "Supergrupos Nacionales": el de Guayaquil (que correspondería aproximadamente a los 10 "imperios" de Hanson y el de la
Filantrópica (que correspondería a la "oposición libanesa" de Hanson).

El "Supergrupo de Guayaquil" estaría integrado por los sub-grupos Arosemena, Icaza, Estrada, Noboa, Marcos, Carbo, Vallarino, Febres Cordero, Alvarado y controlaba 72 empresas anónimas, 2 empresas de seguros, 7 bancos, y mantenía vínculos con capitales extranjeros.

El Supergrupo La Filantrópica incluía las familias Isaías, Dassum, Kronfle, Baracat, Baccach, Raad, Anton, Adum, Salem, Abbud, Bucaram, y controlaba 53 compañías anónimas, entre inmobiliarias, comerciales, importadores, textiles, automotrices, tres bancos nacionales (La Filantrópica, Banco del Pichincha, Banco Popular) y dos compañías de seguros nacionales.

Otros grupos que Navarro consideraba nacionales son:

- Gorelik (controlaba la producción y distribución de bebidas gaseosas).

- Granda (presente en múltiples sectores, entre ellos agropecuario, industrial, comercial, transporte, inmobiliario, financiero y medios de comunicación).

- Cobo Martínez (Pichincha y Tungurahua).

- Briz (Pichincha y Guayas).

- Dalmau (Pichincha y Guayas).

- Pienknagura (Pichincha y Guayas). 
- Ribadeneira (ADELCA, ECASA y Fosforera Ecuatoriana).

Y 15 grupos provinciales (Ortega, Bozzo, Maspons, Bjarner, Umpiérrez, Zevallos, Dager Bitar, Del Hierro Moran, Encalada Mora, Cuesta Holguín, Gallegos, Malo, Vásquez y Cordero Crespo).

Ya para el trabajo que publiqué con CEDIS en 1986, había identificado 35 grupos económicos, que sumaban 538 empresas, y un capital de 33 mil millones de sucres (21,7\% del total nacional). Se debe considerar que las sucursales y subsidiarias de empresas multinacionales alcanzaba el 21,1\% del capital total, y el Estado 39,8\% del total, por lo cual entre los tres actores sumaban la mayor parte del capital invertido en el país.

En mi estudio publicado por CEDEP en 1991 (que, como he dicho, era una versión revisada y editada de mi Tesis de Grado), opté por separar algunos de los subgrupos incluidos por Navarro en el "Supergrupo de Guayaquil", e identifiqué algunos grupos adicionales.

En total, identifiqué a 52 grupos económicos, que para 1986 controlaban 774 empresas productivas y de servicios y 87 entidades financieras, para un total de 861 empresas. En dicho año, las 774 empresas productivas controlaban el $41,6 \%$ del total de ventas y el $43,1 \%$ del total de capital de todas las empresas productivas y de servicios del país; en tanto que las 87 entidades financieras concentraban el $76 \%$ del crédito y el $72 \%$ del capital invertido en el sistema financiero. Las 861 empresas representaban el 47,9\% del capital total nacional, y, al mismo tiempo, habían recibido el $54,9 \%$ del total de inversión extranjera del país.

Aquí cabe anotar que una parte de la "inversión extranjera" eran capitales de los mismos grupos familiares que se encontraba en paraísos fiscales; mientras que, en otros casos, sobre todo en la industria, se trataba efectivamente de una sociedad entre capitales nacionales y extranjeros.

También cabe anotar que se excluyó de este cálculo el capital de las empresas estatales, tanto en el sector productivo y de servicios, como del sistema financiero.

El SRI, por su parte, comenzó por identificar 17 grupos económicos en 2007, llegando a 100 en el 2012, y 200 en el 2016. Es probable que esto no significase que el número de grupos económicos se ha expandido rápidamente en ese período, sino que el SRI ha profundizado en la identificación de vínculos empresariales.

\section{Empresas del Estado}

En el cuadro 107 de mi libro (1991, pp. 581-583) listaba 76 empresas del Estado (en seis de ellas participaba 
también el capital extranjero, como socio minoritario). Era un listado parcial, dado que solo incluía las compañías de economía mixta y sociedades anónimas en las cuales el Estado tenía una participación preponderante; así como las instituciones financieras estatales. No se incluyeron múltiples empresas estatales, regionales, municipales, así como otras entidades públicas cuya finalidad principal no era la producción de bienes y servicios, aunque los generasen de manera secundaria.

Tampoco se incluían empresas estatales no sujetas a la Superintendencia de Compañías o la Superintendencia de Bancos, reseñadas en las pp. 579-580 del libro, entre ellas:

- Siete empresas adscritas al Ministerio de Defensa, incluyendo FLOPEC, TAME y Transnave.

- Tres empresas adscritas al Ministerio de Energía: CEPE (hoy Petroecuador y sus filiales), INECEL e INEMIN.

- Seis empresas adscritas al Ministerio de Agricultura, incluyendo ENAC, ENPROVIT, y la Empresa Nacional de Semen (ENDES).

- Tres empresas adscritas al Ministerio de Industrias: Empresa de Alcoholes del Estado, Empresa Pesquera Nacional y OCEPA.

- Tres empresas del Ministerio de Educación: DINACE, ENPRODE y SNALME.
- Tres empresas del Ministerio de Obras Públicas: IETEL, Correos y Ferrocarriles del Estado.

- El IESS, el IEOS (Obras Sanitarias), la Empresa de Suministros del Estado, las empresas eléctricas regionales, y las empresas municipales de agua potable, alcantarillado, rastro, transporte urbano, etc.

Para 1986, CEPE tuvo ingresos por 67 mil millones de sucres; INECEL por 14 mil millones; IESS por 47 mil millones; $y$ el BEDE (hoy Banco del Estado) por 8,7 mil millones. Estos rubros se comparan con los ingresos del Gobierno Central por 197 mil millones, y un total de ingresos del sector público consolidado por 403 mil millones.

Para dicho año, el sector publico participó en el 18,2\% de la producción bruta de bienes y servicios; $19,8 \%$ del valor agregado bruto; $51 \%$ de las remuneraciones; $35,1 \%$ de la renta de la propiedad; $36,6 \%$ de los impuestos netos, y el 40,1\% de la inversión (formación bruta de capital fijo). Para fines de las Cuentas Nacionales, el sector público estaba constituido por las empresas públicas no financieras, las instituciones financieras públicas y las administraciones públicas.

En la p. 585 de mi libro (1991) se indica la siguiente evolución de la participación del sector público en la producción bruta de bienes y servicios: 
- $\quad$ 10,6\% en 1973 (al iniciarse la producción petrolera),

- $\quad$ 15\% en 1976 (al aumentar la participación de CEPE en el consorcio con Texaco),

- 22,1\% en 1982 (luego de constituirse varias empresas mixtas, así como el desarrollo de empresas eléctricas).

- $\quad$ 18,2\% en 1986, posiblemente como reflejo de la privatización de algunas empresas estatales.

Las instituciones financieras públicas, por su parte, participaron en 1986 con el $59,6 \%$ de los préstamos otorgados, y el 43,2\% de la renta de la propiedad (intereses y otros ingresos) del sector financiero. En 1977, estas relaciones fueron del $56 \%$ y $53,3 \%$, respectivamente.

Tres entidades mantenían participaciones accionarias en 1986: la Corporación Financiera Nacional (CFN) tenía inversiones en 46 empresas activas y seis en liquidación, por un monto total de 5.497 millones (monto que le hubiera colocado tercero entre los grupos económicos privados); el Banco Nacional de Fomento tenía inversiones en 19 empresas, por un total de 1.232 millones; y la Dirección de Industrias del Ejercito (DINE) controlaba ocho empresas y participaba en cuatro adicionales. También se constituyó en 1988 el Banco General Rumiñahui y la compañía de seguros del mismo nombre, con un capital inicial de 1.000 millones (comparado con un capital de 2.597 millones del mayor banco privado de la época, el Banco del Pacífico, en ese entonces parte del grupo Maspons-Laniado).

En la actualidad, el conglomerado empresarial más grande del país sigue siendo el del Estado. Conforme a la Constitución de Montecristi, el Estado no podía ser "marginado" de los "recursos estratégicos", que incluyen "la energía en todas sus formas, las telecomunicaciones, los recursos naturales no renovables, el transporte y la refinación de hidrocarburos, la biodiversidad y el patrimonio genético, el espectro radioeléctrico, el agua, y los demás que determine la ley" (Constitución de la República del Ecuador, 2008, Art. 313).

La mayor empresa del Ecuador es la Empresa Pública Petroecuador; pese al colapso del precio del petróleo, en el 2015 tuvo ingresos por $\$ 9.284$ millones, $y$ utilidades por $\$ 751$ millones; los ingresos son 4,66 veces los ingresos de la mayor empresa privada, Corporación Favorita. Entre las 50 mayores empresas, constan ocho empresas públicas (Petroecuador, CNEL, CNT, CELEC, FLOPEC, EEQ, TAME y Operaciones Río Napo; así como la empresa mixta Omnibus BB). Las ocho empresas sumaron ingresos por \$13.895 millones, cerca del 14\% del PIB. Según la Revista Vistazo (Cavagnaro, 2016), en el 2015 las empresas públicas represen- 
taron el $21 \%$ de los ingresos del listado de las 500 principales empresas, una reducción de cuatro puntos con respecto al 25\% del 2014.

Según una publicación de SENPLADES (2013), "En la actualidad, el Ecuador cuenta con 28 empresas de la Función Ejecutiva, de las cuales 25 son públicas —es decir, 100\% de propiedad estatal - y tres empresas son subsidiarias de economía mixta, donde el Estado tiene al menos el $51 \%$ de participación accionaria. Con respecto a las empresas creadas por los GAD, hasta octubre de 2013 existen alrededor de 212 empresas. En el caso de las universidades se cuenta con diez empresas públicas a nivel nacional" (p. 41). 


\section{Cuadro 1. Empresas públicas de la Función Ejecutiva}

\begin{tabular}{|c|c|}
\hline \multicolumn{2}{|r|}{ SECTORES ESTRATÉGICOS } \\
\hline Hidrocarburos & $\begin{array}{l}\text { EP Petroecuador } \\
\text { Petroamazonas EP } \\
\text { Flota Petrolera Ecuatoriana FLOPEC EP } \\
\text { Refinería del Pacífico CEM } \\
\text { Operaciones Río Napo CEM }\end{array}$ \\
\hline Minería & $\begin{array}{l}\text { Empresa Nacional Minera ENAMI EP } \\
\text { Gran Nacional Minera Mariscal Sucre CEM }\end{array}$ \\
\hline Electricidad & $\begin{array}{l}\text { Corporación Eléctrica del Ecuador CELEC EP } \\
\text { Corporación Nacional de Electricidad CNEL EP } \\
\text { Eléctrica de Guayaquil EP } \\
\text { Cocasinclair EP } \\
\text { Hidrolitoral EP }\end{array}$ \\
\hline Telecomunicaciones & $\begin{array}{l}\text { Corporación Nacional de Telecomunicaciones CNT EP } \\
\text { Televisión y Radio de Ecuador EP RTV Ecuador } \\
\text { Correos del Ecuador EP }\end{array}$ \\
\hline & Ecuador Estratégico EP \\
\hline \multicolumn{2}{|r|}{ Otros Sectores } \\
\hline Productivo & $\begin{array}{l}\text { Tame EP } \\
\text { Ferrocarriles del Ecuador EP } \\
\text { Empresa Pública Cementera del Ecuador EPCE } \\
\text { Infraestructuras Pesqueras del Ecuador EP } \\
\text { Unidad Nacional de Almacenamiento EP } \\
\text { Empresa Pública Importadora EPI EP }\end{array}$ \\
\hline Seguridad & $\begin{array}{l}\text { Astilleros Navales del Ecuador ASTINAVE EP } \\
\text { Empresa de Municiones Santa Bárbara EP } \\
\text { Fabricamos Ecuador FABREC EP }\end{array}$ \\
\hline Social & $\begin{array}{l}\text { Empresa Nacional de Fármacos ENFARMA EP } \\
\text { Empresa Pública de Parques Urbanos y Espacios Públicos }\end{array}$ \\
\hline $\begin{array}{l}\text { Conocimiento y } \\
\text { talento humano }\end{array}$ & Yachay EP \\
\hline
\end{tabular}

Fuente y elaboración: SENPLADES, CGEP 
La CFN, el BNF e ISSFA mantienen, además, acciones en empresas; conforme a un artículo publicado en "El Telégrafo", el ISSFA tiene acciones en 150 empresas, incluyendo 13 que pertenecían previamente al Holding DINE: Fabrilfame, Cossfa, Santa Bárbara, Andec, Hdineagros, Explocen, Aychapicho Agros, Sepriv, Energyhdine y Nergyhdine; las filiales son Soccasa, Omnibus Botar, Banco General Rumiñahui y Condormining (https://goo.gl/rKqCpf).

Conforme al SRI, el Holding DINE (ahora de propiedad del ISSFA) incluye 21 empresas, con activos totales por $\$ 2.133$ millones, ingresos por $\$ 261$ millones, y patrimonio neto por $\$ 1.670$ millones.

\section{Cuadro 2. Grupo DINE / ISSFA. Principales empresas}

\begin{tabular}{|c|c|c|c|}
\hline Razón Social & Provincia & Total Ingresos & Patrimonio Neto \\
\hline $\begin{array}{l}\text { ACERÍAS NACIONALES DEL ECUADOR SOCIEDAD } \\
\text { ANÓNIMA A.N.D.E.C. }\end{array}$ & GUAYAS & 142.386 .591 & 118.296.281 \\
\hline $\begin{array}{l}\text { LA CUADRA COMPAÑÍA INMOBILIARIAY Y COMER- } \\
\text { CIALIZADORA S.A. INMOSOLUCIÓN }\end{array}$ & PICHINCHA & 31.886 .272 & 2.277 .575 \\
\hline EXPLOCEN C.A. & PICHINCHA & 19.932 .366 & 7.524 .451 \\
\hline EMSAAIRPORT SERVICES CEM & PICHINCHA & 18.502 .245 & 13.772 .981 \\
\hline $\begin{array}{l}\text { AMAZONASHOT HOTELERÍA ORGANIZACIONESY } \\
\text { TURISMO S.A. }\end{array}$ & PICHINCHA & 16.883 .131 & 30.456 .013 \\
\hline FABRILFAME S.A. & PICHINCHA & 15.340 .409 & 4.713 .908 \\
\hline $\begin{array}{l}\text { HOLDINGDINE S.A. CORPORACIÓN INDUSTRIALY } \\
\text { COMERCIAL EN LIQUIDACIÓN }\end{array}$ & PICHINCHA & 2.510 .862 & 65.312 .090 \\
\hline INAMAZONAS INMOBILIARIA AMAZONAS S.A. & PICHINCHA & 1.348 .988 & 15.434 .301 \\
\hline $\begin{array}{l}\text { INSTITUTO DE SEGURIDAD SOCIAL DE LAS } \\
\text { FUERZAS ARMADAS }\end{array}$ & PICHINCHA & 383.790 & 1.409.272.809 \\
\hline 21 Empresas & & 261.432 .558 & 1.670 .314 .753 \\
\hline
\end{tabular}

Fuente: SRI

Aparte de las empresas produc- de medios de comunicación (actualmentivas y comerciales, el Estado también es te, es el único actor económico que puede propietario de instituciones financieras y legalmente participar en los tres rubros). 
Las entidades financieras del Estado son:

- Banco del Instituto Ecuatoriano de Seguridad Social (BIESS)

- Banco Ecuatoriano de la Vivienda (BEV)

- $\quad$ Instituto de Fomento al Talento Humano

- Corporación Financiera Nacional (CFN)

- $\quad$ Banco Nacional de Fomento (BNF)

- Banco de Desarrollo del Ecuador (antiguo BEDE).

Aquí no se incluyen al Banco del Pacifico, que anteriormente fue privado y que actualmente pertenece al Estado (junto a su grupo financiero); ni tampoco a COFIEC (otrora cabeza de un poderoso grupo económico, ahora convertido en agencia de cobranzas); la Corporación Nacional de Finanzas Populares y Solidarias (Conafips); la Compañía de Seguros Sucre; Banco General Rumiñahui (otro banco comercial, de capital mixto); o al propio Banco Central del Ecuador (que cumple funciones específicas dentro del sistema monetario y financiero).

Conforme al artículo en Revista Gestión de Brito et al. (junio/julio 2016), el Grupo Financiero Pacífico está actualmente integrado por cuatro entidades: Banco del Pacífico Ecuador, la almacenadora ALMAGRO, Overseas y la subsidiaria del Banco del Pacífico en Panamá. En
1986, cuando todavía era privado, incluía además a Pacific National Bank de Miami; Financiera Ecuatoriana del Pacifico; Unicredit (Mastercard), la Compañía Nacional de Seguros Sucre, y Leasing del Pacifico. El mismo artículo indica que el Grupo Financiero del Pacífico era el segundo en activos, patrimonio y utilidades en el 2015 (\$570 millones en patrimonio, \$56 millones en utilidades en diciembre 2015).

Tampoco se considera en la publicación de SENPLADES el conglomerado de medios de comunicación del Estado, que actualmente incluye:

- Cuatro canales de Televisión: ECTV, Gamavisión, TC Televisión y Cablenoticias.

- $\quad$ El servicio de televisión de pago TV Cable (que a su vez tiene dos canales propios).

- Tres periódicos: El Telégrafo, El Ciudadano (medio digital e impreso), Diario PP.

- Radio Pública del Ecuador (RPE), Radio Casa de la Cultura, radioemisoras TC Radio, Carrusel, Super K, Multicom, América Visión, Organización Radial.

- Agencia Nacional de Noticias de Ecuador y Sudamérica (Andes).

- Revistas La Otra, La Onda, Más, El Agro, Revista Samborondón, y otras revistas de agronomía y farándula. 


\section{Grupo Financiero Pichincha / Diners}

Para la Superintendencia de Bancos, el mayor grupo financiero es el Grupo Pichincha; y en cuarto lugar se encuentra el Grupo Diners (la SB los trata como dos grupos separados; en tanto que el SRI considera que conforman un solo grupo).

Actualmente, según la Superintendencia de Bancos y Brito et al. (2016), el Grupo Pichincha está compuesto por 13 entidades financieras, cuya cabeza es el propio Banco del Pichincha; participaciones (derechos fiduciarios/convenio) con el Banco General Rumiñahui y el Banco de Loja; subsidiarias/filiales en Colombia, Perú, Panamá y España; una agencia en Miami; y las empresas Almesa, Pichincha Sistemas Acovi, Amerafin, Credife, y Vaserum. Es el principal grupo financiero, con activos totales por \$ 13.187 millones, patrimonio por $\$ 1.043$ millones y utilidades por $\$ 56$ millones (a diciembre 2015). Totoy y Vizuete (2012) indican que los tres bancos concentraban un tercio de los ingresos del sistema bancario en el 2010 (p. 80).

En 1986, había incluido en el Subgrupo Pichincha, aparte del Banco, a Banco del Pichincha Limited (Bahamas) y Metropolitana de Seguros; $y$, en el sector real, estaba estrechamente vinculado con el grupo maderero que preside Con- to Patiño (FORESA, INCARMA, Industrias Maestro, Comercial RECORSA, NOVATEX y Empresa Pasteurizadora Quito).

Por su parte, el Grupo Financiero Diners, conforme a la SB, está constituido por siete entidades: Diners Club del Ecuador, C.A.; Interdin S.A.; participación en el Banco del Pichincha Colombia; participación en Pichincha Sistemas Acovi; Datafast; Banred; y el mismo Banco del Pichincha. Para diciembre 2015, tenía activos por $\$ 1.595$ millones, patrimonio por $\$ 278$ millones, y utilidades por $\$ 40$ millones.

En 1986, habíamos incluido en el subgrupo Diners, aparte de Diners Club, a Dinediciones S.A. (empresa posteriormente vendida a sus empleados), Dinformatica, Dinviajes, y Autos Delta. Para el SRI, el Grupo Económico del Banco del Pichincha incluye también a Diners Club, así como a AIG-Metropolitana de Seguros, Interdin y un total de 161 empresas. En el 2015, indica que el grupo (así definido) tuvo ingresos totales por \$1.901 millones, y pagó impuesto a la renta por $\$ 44,2$ millones (2,33\%). El SRI lo considera primero en el ranking de grupos económicos.

En el siguiente cuadro, aparecen sus 16 principales empresas, de un total de 161 empresas vinculadas. 


\section{Cuadro 3. Grupo Económico Pichincha / Diners. Principales empresas}

\begin{tabular}{|c|c|c|c|}
\hline Razón Social & Provincia & Total Ingresos & Patrimonio Neto \\
\hline BANCO PICHINCHACA & PICHINCHA & 1.132 .450 .487 & 882.978 .663 \\
\hline $\begin{array}{l}\text { DINERS CLUB DEL ECUADOR S. A. SOCIEDAD } \\
\text { FINANCIERA }\end{array}$ & PICHINCHA & 287.074 .479 & 278.400 .390 \\
\hline $\begin{array}{l}\text { AIG METROPOLITANA CÍA. DE SEGUROSY } \\
\text { REASEGUROS S.A. }\end{array}$ & PICHINCHA & 168.205 .959 & 32.351 .401 \\
\hline BANCO GENERAL RUMIÑNAHUI S. A. & PICHINCHA & 79.174 .458 & 50.239 .220 \\
\hline $\begin{array}{l}\text { INTERDIN S.A. EMISORA Y ADMINISTRADO- } \\
\text { RA DE TARJETAS DE CRÉDITO }\end{array}$ & PICHINCHA & 66.541 .244 & 52.361 .781 \\
\hline BANCO DE LOJASA & LOJA & 45.664 .338 & 42.905 .368 \\
\hline $\begin{array}{l}\text { CREDIFE DESARROLLO MICROEMPRESARIAL } \\
\text { S.A. }\end{array}$ & PICHINCHA & 28.030 .790 & 4.013 .372 \\
\hline PICHINCHA SISTEMAS ACOVI CA & PICHINCHA & 21.590 .918 & 9.935 .331 \\
\hline BANRED S. A. & GUAYAS & 10.526 .068 & 5.032 .909 \\
\hline OPERACIÓN Y NEGOCIOS NEGCORPBIS S.A. & PICHINCHA & 9.278 .570 & 47.194 .408 \\
\hline AMERAFIN S.A. & PICHINCHA & 5.592 .177 & 1.438 .126 \\
\hline OLEAGINOSAS DEL PUERTO OLIPUERTO S.A. & PICHINCHA & 4.910 .663 & 3.487 .254 \\
\hline VASERUM SA & PICHINCHA & 4.740 .390 & 5.031 .172 \\
\hline ALMACENERA DEL ECUADOR S.A. ALMESA & GUAYAS & 4.420 .150 & 13.847 .552 \\
\hline \multicolumn{2}{|l|}{161 Empresas } & 1.901 .133 .989 & 2.665.779.210 \\
\hline
\end{tabular}

Fuente: SRI (2016)

Conforme al SRI, el grupo eco- $\$ 1.901$ millones; y un patrimonio neto nómico tenía un total de activos por total de $\$ 2.665$ millones.

$\$ 13.432$ millones; total de ingresos por 
Grupo OCP, Repsol, SINOPEC, CNPC

De acuerdo al SRI, el segundo grupo económico más grande es un consorcio integrado, principalmente, por empresas chinas y españolas, en el rubro de hidrocarburos.

Hace 30 años, tales empresas no tenían actividades en el Ecuador (y, además, en mi libro, me enfoqué principalmente en grupos económicos nacionales).

Conforme al SRI, el grupo económico tenía un total de 57 empresas, con ingresos por $\$ 1.835$ millones; total de activos por $\$ 3.881$ millones; $y$ un patrimonio neto total de $\$ 1.673$ millones.

\section{Cuadro 4. Grupo Económico OCP, Repsol, SINOPEC, CNPC. Principales empresas}

\begin{tabular}{|c|c|c|c|}
\hline Razón Social & Provincia & Total Ingresos & Patrimonio Neto \\
\hline ANDES PETROLEUM ECUADOR LTD. & PICHINCHA & 588.046 .551 & 560.630 .474 \\
\hline CONSORCIO PETROLERO BLOQUE 16 & PICHINCHA & 209.281.009 & 392.284.305 \\
\hline OLEODUCTO DE CRUDOS PESADOS (OCP) ECUADOR S.A. & PICHINCHA & 207.852 .447 & $(145.704 .636)$ \\
\hline REPSOL ECUADORS.A. & PICHINCHA & 139.737 .506 & 80.679 .509 \\
\hline CONSORCIO PETROLERO BLOQUE 17 & PICHINCHA & 118.853 .248 & 24.718 .712 \\
\hline DURAGASS.A. & GUAYAS & 100.615 .550 & 8.263 .708 \\
\hline $\begin{array}{l}\text { SINOPEC INTERNATIONAL PETROLEUM SERVICE ECUADOR } \\
\text { S.A. }\end{array}$ & $\mathrm{PICHINCHA}$ & 95.492 .859 & 65.901 .344 \\
\hline OVERSEAS PETROLEUM AND INVESTMENT CORPORATION & $\mathrm{PICHINCHA}$ & 95.192 .703 & 421.225 .839 \\
\hline $\begin{array}{l}\text { CNPC CHUANQING DRILLING ENGINEERING COMPANY } \\
\text { LIMITED }\end{array}$ & PICHINCHA & 87.137 .491 & 14.237 .839 \\
\hline PETROORIENTAL S.A. & PICHINCHA & 78.365 .799 & 59.698 .645 \\
\hline AMODAIMI OIL COMPANY, S.L. & PICHINCHA & 72.266 .464 & 205.858 .549 \\
\hline CONSORCIO PETROLERO BLOQUETIVACUNO & PICHINCHA & 35.511 .485 & 12.729 .877 \\
\hline 57 Empresas & & 1.835 .323 .671 & 1.673.254.172 \\
\hline
\end{tabular}

Fuente: SRI (2016) 


\section{Grupo Eljuri / Banco del Austro}

Es sin duda uno de los grupos que más se han consolidado en los últimos años, constituyéndose en la actualidad en el tercer grupo económico más importante del país, conforme al SRI (2016).

Incluye un total de 327 entidades, con un total de ingresos por $\$ 1.868 \mathrm{mi}$ llones; activos por\$3.998 millones; y patrimonio neto por $\$ 1.033$ millones.

La Superintendencia de Bancos lo clasifica como Grupo Financiero Austro, e incluye al Banco del Austro, Almacopio y Financiera del Austro (FIDASA). El SRI incluye en el grupo a otras entidades financieras, entre ellas Seguros Unidos. También mantiene inversiones en medios de comunicación, incluyendo Telerama y Telecuador.

En 1986, el Subgrupo Eljuri (lo había clasificado como parte del Grupo del Azuay) incluía 10 empresas productivas y comerciales (destacándose los Almacenes Juan Eljuri y varias ensambladoras de motocicletas), y cuatro entidades financieras (Banco del Austro, FIDASA, Cambiazuay y Alianza Cía. De Seguros). Varias de las empresas vinculadas a la ce-
rámica(Ecuaceramica, Cerámica Andina, Cerámica Rialto, Keramikos) las había clasificado como parte del Grupo Chiriboga Vollmer (vinculado a su vez a inversionistas venezolanos); en tanto que varias empresas vinculadas a la industria automotriz y lubricantes las había incluido en el Subgrupo CEPSA, parte del Grupo PROINCO (entre ellas AYMESA, Metrocar, Anglo Ecuadorian). No está claro si el Grupo Eljuri siempre tuvo participación en dichas empresas, o adquirió paquetes accionarios en años recientes.

Doce entidades del grupo están domiciliadas en paraísos fiscales, y 41 entidades o personas aparecieron vinculadas en los "Panamá Papers", de acuerdo al SRI. En 2011, Juan Eljuri viajó con el Presidente Correa en una delegación a Corea del Sur, para promover la inversión de Hyundai en una ensambladora en Manta (Pástor, 2016, p. 64). Totoy y Vizuete (2012) indican que el Grupo Eljuri controla el 87\% del CIIU 2691 (Fabricación de otros productos de porcelana y de cerámica), y 74\% del CIIU 2693 (Fabricación de productos de cerámica para uso estructural). 


\section{Cuadro 5. Grupo Económico Eljuri / Austro. Principales empresas}

\begin{tabular}{|c|c|c|c|}
\hline Razón Social & Provincia & Total Ingresos & Patrimonio Neto \\
\hline AEKIA S.A. & PICHINCHA & 185.072 .230 & 73.524 .930 \\
\hline BANCO DEL AUSTROS. A & AZUAY & 166.931 .031 & 145.411 .454 \\
\hline NEGOCIOS AUTOMOTRICES NEOHYUNDAI S.A. & AZUAY & 161.088 .587 & 106.756.874 \\
\hline ALMACENES JUAN ELUURI CIA. LTDA. & AZUAY & 118.746 .084 & 25.400 .111 \\
\hline AYMESA S.A. & PICHINCHA & 113.094 .540 & 65.365 .331 \\
\hline METROCARS.A. & AZUAY & 113.066 .797 & 21.743 .081 \\
\hline QUITO MOTORS S.A. COMERCIAL E INDUSTRIAL & AZUAY & 89.970 .524 & 32.104 .830 \\
\hline ASIAUTOS.A & PICHINCHA & 83.336 .046 & 19.021.778 \\
\hline KMOTORS.A. KMOT & GUAYAS & 43.368 .065 & 8.948 .912 \\
\hline KERAMIKOS S.A. & AZUAY & 41.650 .125 & 16.304 .101 \\
\hline C A ECUATORIANA DE CERÁMICA & AZUAY & 39.586 .468 & 74.356 .590 \\
\hline LAS FRAGANCIAS CIA. LTDA. & AZUAY & 35.094 .316 & 25.025 .330 \\
\hline CERÁMICA RIALTOS.A. & AZUAY & 34.387 .273 & 47.904 .259 \\
\hline COMPAÑÍA NACIONAL DE GAS CONGAS C.A. & $\mathrm{PICHINCHA}$ & 33.498 .647 & 11.288 .626 \\
\hline FISUM S.A. & AZUAY & 33.068 .832 & 6.944 .282 \\
\hline SEGUROS UNIDOS S.A. & PICHINCHA & 32.353 .838 & 15.187.677 \\
\hline ITALPISOS SA & AZUAY & 27.329 .051 & 16.267.219 \\
\hline 327 Empresas & & 1.868.557.735 & 1.033 .159 .923 \\
\hline
\end{tabular}

Fuente: SRI (2016)

\section{Grupo Wright / Favorita}

Es otro grupo que se ha venido consolidando en las últimas décadas. En 1986, lo identificábamos como Grupo PROINCO, y le asignábamos un total de 34 empresas, con 14,1 mil millones de sucres en ventas. En aquel entonces, estaba íntimamente imbricado con el Banco de la Producción, y las entida- des financieras PROINCO, Casa Paz y Seguros Equinoccial. Recientemente, con las normas legales que requerían la separación de las instituciones financieras de las empresas del sector real, el Produbanco fue primero desvinculado del grupo, y posteriormente vendido al banco centroamericano Promérica. 
De acuerdo al SRI, en la actualidad el grupo Corporación Favorita está integrado por 119 empresas, entre las cuales se destaca la empresa Corporación Favorita, dueña de los supermercados (SuperMaxi, MegaMaxi, Aki), siendo la mayor empresa privada del país, con ventas por $\$ 1.989$ millones, y utilidades por $\$ 146$ millones en el 2015. Su contrincante más cercano, Corporación El Rosado (Mi Comisariato) tuvo ventas por $\$ 1.044$ millones, y utilidades por $\$ 22$ millones. Totoy y Vizuete (2012) estiman que en el 2010 Corporación Favorita concentraba el 31,8\% del CIIU G513. Las 119 empresas sumaron activos totales por $\$ 2.120$ millones, ingresos por $\$ 2.695$ millones, y un patrimonio neto de $\$ 1.566$ millones.

\section{Cuadro 6. Grupo Wright / Favorita. Principales empresas}

\begin{tabular}{|c|c|c|c|}
\hline Razón Social & Provincia & Total Ingresos & Patrimonio Neto \\
\hline CORPORACIÓN FAVORITA C.A. & PICHINCHA & 1.989.012.100 & 1.013.208.037 \\
\hline COMERCIAL KYWIS.A. & PICHINCHA & 276.884 .992 & 139.670 .474 \\
\hline COMOHOGARSA & PICHINCHA & 100.937.798 & 90.963 .906 \\
\hline TELEVISIÓN Y VENTAS TELEVENTS.A. & PICHINCHA & 65.739 .158 & 23.689 .054 \\
\hline FLEXIPLAST S.A. & PICHINCHA & 54.524 .684 & 31.218 .292 \\
\hline POLLO FAVORITOSA POFASA & PICHINCHA & 40.612 .268 & 27.627 .859 \\
\hline BEBELANDIA S.A. & PICHINCHA & 23.483 .837 & 16.726 .557 \\
\hline MAXIPAN S.A. & PICHINCHA & 14.408 .605 & 8.475 .592 \\
\hline AGROPESA INDUSTRIA AGROPECUARIA ECUATORIANA SA & PICHINCHA & 13.111 .900 & 12.645 .001 \\
\hline ALIMENTOS Y SERVICIOS ECUATORIANOS ALISERVIS S.A. & PICHINCHA & 11.353 .521 & 2.866 .157 \\
\hline INVERSIÓNY YESARROLLO INVEDES.A. & PICHINCHA & 11.289 .234 & 48.634 .145 \\
\hline ENERMAXS.A & PICHINCHA & 9.712 .556 & 23.553 .055 \\
\hline MISTERBOOKS S.A. & PICHINCHA & 9.233 .304 & 3.815 .748 \\
\hline AUDIOAUTOS.A. & PICHINCHA & 9.151 .848 & 6.098 .102 \\
\hline IMPORPOINT S.A. & GUAYAS & 6.464 .532 & 7.490 .603 \\
\hline \multicolumn{2}{|l|}{119 Empresas } & 2.695.673.978 & 1.566 .612 .034 \\
\hline
\end{tabular}

Fuente: SRI (2016) 


\section{Grupo Schlumberger}

El siguiente grupo de acuerdo al SRI es Schlumberger, un grupo de empresas de servicios petroleros extranjeras. El grupo está constituido por 29 empresas, de las cuales las dos principales, el Consorcio Shushufindi y Schlumberger del Ecuador, corresponden al grueso de ingresos y patrimonio. En 1986 no apa- recía en el listado de grupos. El Consorcio Shushufindi fue en 2015 la tercera empresa privada más grande del país, con $\$ 516$ millones en ventas y $\$ 160$ mi\|lones en utilidades. Las 29 empresas del grupo tienen $\$ 2.747$ millones en activos, $\$ 996$ millones en ingresos y $\$ 1.438$ millones en patrimonio neto.

\section{Cuadro 7. Grupo Schlumberger. Principales empresas}

\begin{tabular}{|l|r|r|r|}
\hline Razón Social & Provincia & Total Ingresos & Patrimonio Neto \\
\hline CONSORCIO SHUSHUFINDI S.A. & PICHINCHA & 516.151 .905 & 511.326 .706 \\
\hline SCHLUMBERGER DEL ECUADOR S.A. & PICHINCHA & 448.562 .842 & 325.412 .274 \\
\hline SAXON ENERGY SERVICES DEL ECUADOR S.A. & PICHINCHA & 15.593 .743 & 15.682 .652 \\
\hline KAMANA SERVICES S.A. & PICHINCHA & 9.757 .526 & 62.198 .001 \\
\hline SCHLUMBERGER SURENCO S.A. & PICHINCHA & 5.953 .212 & 218.065 .957 \\
\hline 29 Empresas & \multicolumn{3}{|c|}{} \\
\hline
\end{tabular}

Fuente: SRI (2016)

\section{Grupo CLARO (Slim)}

Grupo constituido en torno a CONECEL, operadora de telefonía celular (CLARO/AmericaMovil) del magnate mexicano Carlos Slim. CONECEL es la segunda mayor empresa privada del país, con ventas por $\$ 1.520$ millones y utilidades por \$141 millones en el 2015. Las 22 empresas del grupo tienen activos totales por $\$ 2.079$ millones, ingresos por $\$ 1.607$ millones, patrimonio neto por $\$ 522$ millones, y aportaron al fisco \$324 millones. 


\section{Cuadro 8. Grupo CLARO. Principales empresas}

\begin{tabular}{|c|c|c|c|}
\hline Razón Social & Provincia & Total Ingresos & Patrimonio Neto \\
\hline $\begin{array}{l}\text { CONSORCIO ECUATORIANO DE TELECOMUNICA- } \\
\text { CIONES S.A. CONECEL }\end{array}$ & GUAYAS & 1.540 .225 .902 & 147.016 .999 \\
\hline ECUADORTELECOM S.A. & GUAYAS & 65.176 .843 & 1.769 .946 \\
\hline AMOVECUADORS.A. & PICHINCHA & 1.701 .320 & 373.628 .092 \\
\hline \multicolumn{2}{|l|}{22 Empresas } & 1.607 .282 .562 & 522.412 .151 \\
\hline
\end{tabular}

Fuente: SRI (2016)

\section{Grupo PRONACA (Bakker-Klein- Vilaseca)}

En mi libro (Fierro, 1991) había clasificado a una parte de este grupo como el Subgrupo Bakker, vinculado al Grupo Chiriboga-Vollmer; en tanto que había incluido a los Subgrupos Klein y Vilaseca como parte del ahora desaparecido Grupo COFIEC.

Hacia 1986, el Subgrupo Klein incluía las empresas Industria Cartonera Asociada, Industrias Omega, Tuboplast e Inverconsa; el Subgrupo Vilaseca, la Empacadora Ecuatoriana Danesa (Plumrose), Industria Jabonera Ecuatoriana, Fábrica de Envases (FADESA) e Ind. Metálicas; y el Subgrupo Bakker Aves Andinas, Aves del Pichincha, Aves Procesadas, Incubadora Nacional, INDAVES, PRONACA, INDIA C.A. y Restaurantes Nacionales (Tom Pollo). El Swissotel (H.O.V. Hotelera Quito), por su parte, lo había incluido en el Subgrupo Custer del Grupo Ecuasuiza. Lo cierto es que en compañías anónimas con transacciones en Bolsa varios grupos familiares/económicos pueden participar en una misma empresa; y se pueden configurar los grupos de diferente manera.

En cualquier caso, según la clasificación del SRI, el Grupo PRONACA estaría conformado por 153 empresas, con un total de activos por \$1.546 millones, ingresos por $\$ 1.803$ millones y patrimonio neto por $\$ 830$ millones, montos de los cuales aproximadamente la mitad corresponde a la empresa PRONACA (que fue en 2015 la cuarta empresa privada más grande del país, con ventas por $\$ 995$ millones y utilidades por $\$ 40,7$ millones). Totoy y Vizuete (2012) indica que la empresa concentró el $87 \%$ de los ingresos del CIIU 1511 (Procesamiento y conservación de carne). 


\section{Cuadro 9. Grupo PRONACA. Principales empresas}

\begin{tabular}{|c|c|c|c|}
\hline Razón Social & Provincia & Total Ingresos & Patrimonio Neto \\
\hline $\begin{array}{l}\text { PROCESADORA NACIONAL DE ALIMENTOS } \\
\text { C.A. PRONACA }\end{array}$ & PICHINCHA & 995.818 .210 & 410.601 .944 \\
\hline NOVACEROS.A & PICHINCHA & 222.081 .107 & 84.083 .619 \\
\hline FÁBRICA DE ENVASES S.A. FADESA & GUAYAS & 101.036 .652 & 53.263 .049 \\
\hline $\begin{array}{l}\text { SEAFMAN SOCIEDAD ECUATORIANA DE ALIMENTOSY } \\
\text { FRIGORIFIICOS MANTA CA }\end{array}$ & MANABÍ & 83.696 .773 & 23.956 .437 \\
\hline TROPICALIMENTOS S.A. & GUAYAS & 41.088 .734 & 2.258 .117 \\
\hline I.A.E. INDUSTRIA AGRICOLA EXPORTADORA INAEXPO C.A. & PICHINCHA & 35.764 .656 & 29.282 .592 \\
\hline LA LLAVES.A. DE COMERCIO & GUAYAS & 31.467 .804 & 6.784 .140 \\
\hline FERMAGRI S.A. & PICHINCHA & 29.075 .617 & 5.386 .193 \\
\hline ECUAVEGETAL S. A. & LOS RIOS & 27.909 .386 & 12.523 .378 \\
\hline VECONSAS.A. & GUAYAS & 27.571 .516 & 12.994 .174 \\
\hline ECUAIMCO S.A. & GUAYAS & 27.526 .595 & 5.302 .905 \\
\hline H.O.V. HOTELERA QUITOSA & PICHINCHA & 19.309 .085 & 33.469 .628 \\
\hline AGRÍCOLA OFICIAL S.A. AGROFICIAL & GUAYAS & 18.345 .102 & 5.703 .515 \\
\hline LATIENVASES S.A. & GUAYAS & 17.973 .573 & 9.749 .870 \\
\hline PROLACHIV S.A. & GUAYAS & 14.944 .096 & 3.882 .948 \\
\hline INCUBADORA NACIONAL CA INCA & PICHINCHA & 13.861 .986 & 5.377 .974 \\
\hline \multicolumn{2}{|l|}{153 Empresas } & 1.803 .089 .883 & 830.061 .179 \\
\hline
\end{tabular}

Fuente: SRI (2016)

\section{Grupo Banco de Guayaquil (Lasso)}

El Banco de Guayaquil es actual-

Si bien previamente reportaba mente el tercer grupo financiero más grande (después de los grupos financieros encabezados por los Bancos del Pichincha y Pacífico). Tiene activos por \$3.799 millones, ingresos por \$440 millones, y patrimonio neto por $\$ 634$ millones. como un Grupo Financiero a la Superintendencia de Bancos, en 2015 dejó de hacerlo. Aparte del Banco, el SRI indica que forman parte del grupo la Corporación MultiBG y Promoquil, entre 40 entidades o razones sociales. 
El Banco fue fundado en 1923 con el nombre de Banco Italiano, y en los años 1960s Hanson (1971) lo vinculó a la familia Valdez y Dillon Valdez. No obstante, en 1970 sufrió un colapso, y hubo un salvataje organizado por César Duran Ballén, para entonces vicepresidente de COFIEC. En 1984, tomó control del banco un grupo de accionistas vinculado a FINANSUR, encabezados por Danilo Carrera y Guillermo Lasso. En mi libro, indicaba que la familia Lasso Mendoza participaba también en Industrias Bioacuáticas (INBIOSA), la compañía Laroplast, Constructora e Inmobiliaria Alfa
Omega, FINANSUR y Banunión. Ninguna de estas empresas aparece en el listado del SRI, quizás porque fueron liquidadas o fusionadas; en el caso de FINANSUR, se fusionó con el Banco en 1989. El SRI indica que el grupo tiene relación con el grupo Ecuatoriano Suiza en un fideicomiso, y con el Grupo El Universo en otro fideicomiso. Así mismo, indica que una empresa asociada, Banisi S.A., está constituida en Panamá.

Guillermo Lasso, quien renunció a la Presidencia del Banco, fue candidato presidencial en 2013 y 2017 por su partido (CREO).

\section{Cuadro 10. Grupo Lasso / Banco de Guayaquil. Principales empresas}

\begin{tabular}{|l|c|r|r|}
\hline Razón Social & Provincia & \multicolumn{1}{|c|}{ Total Ingresos } & \multicolumn{1}{|c|}{ Patrimonio Neto } \\
\hline BANCO GUAYAQUIL S.A. & GUAYAS & 423.605 .896 & 401.241 .775 \\
\hline CORPORACIÓN MULIIBG S.A. & GUAYAS & 6.609 .308 & 139.338 .864 \\
\hline FIDEICOMISO MERCANTIL DE ADMINISTRACIÓN GLM & GUAYAS & 1.864 .663 & 18.826 .517 \\
\hline FIDEICOMISO MERCANTIL DE ADMINISTRACIÓN MELM & GUAYAS & 828.800 & 8.451 .919 \\
\hline FIDEICOMISO MERCANTIL DE ADMINISTRACIÓN CLM-MMS & GUAYAS & 705.972 & 7.015 .913 \\
\hline PROMOQUIL S.A. & GUAYAS & 633.092 & 14.743 .222 \\
\hline & & $\mathbf{4 4 0 . 1 0 1 . 2 6 3}$ & $\mathbf{6 3 4 . 4 1 8 . 2 7 4}$ \\
\hline
\end{tabular}

Fuente: SRI (2016)

\section{Grupo ProduBanco (Promérica)}

Como se mencionó anteriormente, ProduBanco estaba vinculado a los grupos PROINCO/Wright/Favorita, hasta que tuvo que desvincularse por motivos legales. Más recientemente, fue vendido al Grupo Banco Promérica, un grupo financiero centroamericano.

Según la Superintendencia de Bancos, es el tercer grupo financiero 
más grande, e incluye, además del banco, a las empresas Exsersa (Servipagos) y Protrámites. El Banco, como tal, era el cuarto mayor del sistema, con activos por $\$ 3.676$ millones, patrimonio por
\$311 millones, yutilidades por \$4 millones. Según el SRI, también aparece vinculado al grupo Accival, varios fideicomisos, y Proamerica Financial Corp. de Panamá.

\section{Cuadro 11. Grupo ProduBanco. Principales empresas}

\begin{tabular}{|l|r|r|r|}
\hline Razón Social & Provincia & Total Ingresos & Patrimonio Neto \\
\hline BANCO DE LA PRODUCCIÓN S.A. PRODUBANCO & PICHINCHA & 317.720 .264 & 311.547 .075 \\
\hline EXTERNALIZACIÓN DE SERVICIOS S.A. EXSERSA & PICHINCHA & 15.480 .812 & 2.757 .028 \\
\hline FIDEICOMISO MERCANTIL PRODUBANCO 3, FIMPROD 3 & PICHINCHA & 2.440 .974 & 1.000 \\
\hline FIDEICOMISO MERCANTIL PRODUBANCO 2 FIMPROD 2 & PICHINCHA & 1.575 .801 & 1.000 \\
\hline $\begin{array}{l}\text { FIDEICOMISO PRIMERA TITULARIZACIÓN } \\
\text { DECARTERA HIPOTECARIA DEVIVIENDA BANCO PROMÉRICA }\end{array}$ & PICHINCHA & 1.335 .526 & 2.899 .704 \\
\hline $\begin{array}{l}\text { ACCIONESYVALORES CASA DEVALORES } \\
\text { S.A. ACCIVAL }\end{array}$ & PICHINCHA & 1.017 .865 & 162.841 \\
\hline \multicolumn{2}{|l|}{} \\
\hline $\mathbf{2 8}$ Empresas
\end{tabular}

Fuente: SRI (2016)

\section{Grupo El Rosado (Czarninski)}

El Grupo Corporación El Rosado (Mi Comisariato, Czarninski) encabeza el otro conglomerado de supermercados en el país. En el 2015, conforme a la Revista Gestión (Brito et al., sept/oct 2016), Corporación El Rosado fue la sexta mayor empresa privada, con ventas por $\$ 1.044$ millones, activos por $\$ 620$ millones, y utilidades por $\$ 22$ millones.

En 1986, aparte de El Rosado, integraban el grupo Alimentos Ecuatorianos
S.A.; Alimentos Superba; y Alimentos del Ecuador C.L. Para el 2015, acorde al SRI, integraban el grupo 68 empresas y razones sociales, incluyendo Truisfruit, Supercines, Panadería del Pacífico, y tres empresas radiales (Entrepasa, Radio Concierto Guayaquil, y Radio Concierto Austro). El total de activos del grupo alcanzaba a \$1.537 millones, los ingresos a $\$ 1.497$ millones, y el patrimonio neto a $\$ 466$ millones (con dos tercios aproximadamente correspondientes a El Rosado). Compartían la em- 
presa Adepasa con el grupo Reybanpac (Wong). Truisfruit se ha convertido en la segunda mayor empresa exportadora de banano, después de UBESA; hasta el 2014, el SRI lo había incluido en el grupo de Álvaro Noboa. Según el SRI, 13 integrantes se encontraban domiciliados en paraísos fiscales, particularmente en Panamá.

\section{Cuadro 12. Grupo El Rosado (Czarninski). Principales empresas}

\begin{tabular}{|c|c|c|c|}
\hline Razón Social & Provincia & Total Ingresos & Patrimonio Neto \\
\hline CORPORACIÓN EL ROSADOS.A. & GUAYAS & 1.051.620.924 & 254.562 .991 \\
\hline TRUISFRUIT S.A. & GUAYAS & 159.851 .158 & $(5.632 .635)$ \\
\hline ADMINISTRADORA DEL PACÍFICO S.A. ADEPASA & GUAYAS & 74.487 .563 & 531.275 \\
\hline SUPERCINESS.A. & GUAYAS & 72.750 .285 & 1.197 .183 \\
\hline INMOBILIARIA MOTKE S.A. & GUAYAS & 41.723 .430 & 66.018 .433 \\
\hline COMDERE S.A. & GUAYAS & 24.289 .624 & 188.938 \\
\hline INMOBILIARIA LAVIE S.A. & GUAYAS & 19.498 .796 & 125.117.109 \\
\hline ENTRETENIMIENTO DEL PACÍFICO S.A. ENTREPASA & GUAYAS & 15.518 .593 & 1.511 .939 \\
\hline SERUVIS.A. & GUAYAS & 9.148 .537 & 729.207 \\
\hline PANADERÍA DEL PACÍFICO S.A. PANPACSA & GUAYAS & 8.190 .903 & 477.096 \\
\hline SUPER PUNTOS S.A. (SPSA) & GUAYAS & 5.946 .275 & 793.165 \\
\hline FRUSHIS.A. & GUAYAS & 3.553 .063 & 1.491 .236 \\
\hline RADIO CONCIERTO GUAYAQUIL S.A. CONCERTQUIL & PICHINCHA & 1.055 .993 & 328.193 \\
\hline INMOBILIARIA MERIDIONAL S.A. INMERISA & GUAYAS & 1.020 .680 & 146.273 \\
\hline RADIO CONCIERTO CONCERT AUSTRO S.A. & PICHINCHA & 82.704 & 26.551 \\
\hline 68 Empresas & & 1.497.973.413 & 466.360 .168 \\
\hline
\end{tabular}

Fuente: SRI (2016)

\section{Grupo Álvaro Noboa (Exportadora Bananera Noboa)}

Nos saltamos del grupo n. 10 (El Rosado) a los grupos n.o 20 (Álvaro Noboa) y 28 (Isabel Noboa), por su relevancia histórica. Los hermanos Noboa Pon- tón fueron los principales herederos de su padre, Luis Noboa Naranjo, quien era considerado hasta su fallecimiento como la persona más acaudalada del Ecuador. En medio de disputas por la herencia, finalmente los dos hermanos quedaron al 
frente de sus respectivos grupos.

El Subgrupo Noboa Naranjo poseía, hacia 1986,51 empresas, y con ventas por 40,7 mil millones de sucres, era el grupo económico más poderoso del país en aquella época (cabe recordar que el Presidente entre 1984 y 1988, León Febres Cordero, había sido gerente de varias de las empresas del grupo). Noboa Naranjo dominaba la producción y exportación de banano, y además había incursionado en la producción y exportación de café, cacao, arroz y azúcar. Hacia 1986, el grupo controlaba 22 mil hectáreas de producción agrícola (Fierro, 1991, p. 265). En aquel entonces, aparte de la Exportadora Bananera Noboa, las principales empresas por ventas eran Industrial Molinera, Industria Cartonera Ecuatoriana, Comp. Agrícola La Julia, Agrícola Clementina, Ingenio San Carlos, Colcafé, Ultramares Corporación, Manufactura de Cartón (MACARSA), Distribuidora Dispacific, entre otros.

Para el 2015, el grupo no es ni un pálido reflejo de lo que fue alguna vez. Tres empresas emblemáticas, Exportadora Bananera Noboa, Agrícola Bananera Clementina y MACARSA, aparecen virtualmente quebradas, con ingresos de cero ( $\$ 46$ mil en el caso de MACARSA) y un patrimonio neto exiguo (negativo en el caso de MACARSA). La Hacienda Clementina fue embargada, por no pago de impuestos; y eventualmente entregada a una cooperativa de trabajadores.
Entre las principales empresas productivas se encuentra Elaborados de Café (ELCAFE), Industrial Molinera, Fertilizantes del Pacífico, Industria Cartonera Ecuatoriana, y Distribuidora Dispacific.

El grupo prácticamente se ha desvanecido entre los principales exportadores de banano, aunque mantiene el liderazgo en la producción y exportación de elaborados de café (ELCAFE). Truisfruit, la segunda mayor exportadora de banano, era clasificada por el SRI como parte del Grupo Noboa hasta el 2014, pero en el 2015 lo incluyó en el Grupo El Rosado.

Llama la atención que el SRI incluye en el grupo a varias instituciones financieras: Banco del Litoral, Generali Cía. De Seguros, Seguros Cóndor, y Global Sociedad Financiera (pese a la prohibición legal para que accionistas de instituciones financieras posean otro tipo de empresas). El Banco del Litoral tuvo pérdidas en 2015, y un alto porcentaje de cartera improductiva. El grupo ha enfrentado demandas judiciales, de pago de impuestos y embargos de varias de sus empresas, tanto a nivel del Ecuador como en el mercado internacional. También se enfrentó a sus hermanos por la herencia de su padre.

Las 183 empresas y razones sociales del grupo alcanzaban a \$1.174 millones en activos, $\$ 549$ millones en ingresos y \$208 millones en patrimonio neto. Los ingresos del grupo han venido declinando, de $\$ 826$ millones en el 2013, a $\$ 731$ 
millones en el 2014, y \$549 millones en el ha sido cinco veces candidato a la Presiúltimo año. 17 de sus integrantes están dencia, llegando a la segunda vuelta en domiciliadas en paraísos fiscales. Noboa dos ocasiones.

\section{Cuadro 13. Grupo Álvaro Noboa. Principales empresas}

\begin{tabular}{|c|c|c|c|}
\hline Razón Social & Provincia & Total Ingresos & Patrimonio Neto \\
\hline COMPAÑÍA DE ELABORADOS DE CAFÉ ELCAFE C.A. & MANABÍ & 128.909 .018 & 12.927 .739 \\
\hline INDUSTRIAL MOLINERA CA & GUAYAS & 77.300 .136 & 42.238 .477 \\
\hline FERTILIZANTES DEL PACÍFICO FERPACIFIC S.A. & GUAYAS & 37.820 .340 & 1.158 .298 \\
\hline GENERALI ECUADOR COMPAÑÍA DE SEGUROS S.A. & GUAYAS & 36.924 .855 & 14.728 .217 \\
\hline DISTRIBUIDORA DISPACIFSA & GUAYAS & 35.301 .092 & 1.841 .448 \\
\hline INDUSTRIA CARTONERA ECUATORIANA S.A. & GUAYAS & 32.940 .658 & 6.279 .519 \\
\hline COMPAÑÍA AGRÍCOLA LA JULIA S.A. CALAJUSA & GUAYAS & 29.111 .155 & $4.516,040$ \\
\hline INDUSTRIAL BANANERA ALAMOSSA & GUAYAS & 22.265 .532 & 3.762 .260 \\
\hline COMPAÑÍA DE SEGUROS CÓNDOR S.A. & GUAYAS & 20.080 .284 & 23.304.153 \\
\hline MOLINOS POULTIERS.A. & СОТОРАХI & 13.491 .033 & $8.030,107$ \\
\hline COMPAÑÍA AGRÍCOLA RÍO VENTANAS S. A. CARIVESA & GUAYAS & 10.753 .148 & 56.396 \\
\hline BANANERA LAS MERCEDES S.A. & GUAYAS & 10.266 .001 & 232.598 \\
\hline COMPAÑÍA AGRÍCOLA LOMA LARGA S.A. CALOLASA & GUAYAS & 9.619 .258 & 135.906 \\
\hline LINEAS AÉREAS NACIONALES ECUADOR S.A. LAN - ECUADOR & GUAYAS & 9.577 .746 & 1.727 .055 \\
\hline COMPAÑÍA NACIONAL DE PLÁSTICOS CONAPLAS S.A. & GUAYAS & 7.700 .510 & 13.374.115 \\
\hline CORPORACIÓN AUTOMOTRIZ SOCIEDAD ANÓNIMA & GUAYAS & 5.249 .059 & 2.872 .752 \\
\hline GLOBAL SOCIEDAD FINANCIERA S. A. & GUAYAS & 3.563 .136 & 4.877 .545 \\
\hline BANCO DEL LITORAL S.A. & GUAYAS & 3.283 .232 & 4.070 .414 \\
\hline COMPAÑÍA AGRÍCOLA BANANERA DEL ECUADOR S.A. CABE & GUAYAS & 2.691 .567 & 279.531 \\
\hline EXPORTADORA BANANERA NOBOA S.A. & GUAYAS & - & 611.934 \\
\hline AGRÍCOLA BANANERA CLEMENTINA S.A. & GUAYAS & - & 928.428 \\
\hline MANUFACTURAS DE CARTÓN S.A. MACARSA & GUAYAS & 46.815 & (788.584) \\
\hline 183 Empresas/Razones Sociales & & 549.773.581 & 208.539.534 \\
\hline
\end{tabular}

Fuente: SRI (2016) 


\section{Grupo Isabel Noboa (Corporación NOBIS)}

Se trata del grupo conformado por Isabel Noboa y sus hijos Romero Noboa, como resultado del desprendimiento de algunas empresas del grupo Noboa Naranjo, al concluir el juicio por la herencia de su padre. Su exesposo, Isidro Romero Carbo, fue Vicepresidente del Grupo Noboa Naranjo, quien también presidió Ultramares Corporación y fue director en el extinto Banco de Crédito e Hipotecario.

Las dos principales empresas del grupo son la Azucarera Valdez (heredada de su padre), y La Universal, junto a varias empresas inmobiliarias. En total, el grupo está constituido por 105 empresas, con activos totales por $\$ 902$ millones, ingresos por $\$ 302$ millones y patrimonio neto por $\$ 369$ millones.

Según el SRI, mantiene relaciones con el grupo del Ingenio San Carlos, al ser copropietarios de tres empresas (Carbogas, Cía. Nacional de Melazas, Sociedad de Destilación de Alcoholes); y con el grupo Eurofish en dos empresas (Realnet y Unifelsa). 16 de sus empresas integrantes están domiciliadas en paraísos fiscales. Aparece como Director Ejecutivo del Consorcio NOBIS Roberto Dunn (que encabezaba un grupo en los años 1980s). 


\section{Cuadro 14. Grupo Isabel Noboa (NOBIS). Principales empresas}

\begin{tabular}{|c|c|c|c|}
\hline Razón Social & Provincia & Total Ingresos & Patrimonio Neto \\
\hline COMPAÑÍA AZUCARERA VALDEZ S.A. & GUAYAS & 127.420 .179 & 133.170 .829 \\
\hline UNIVERSAL SWEET INDUSTRIES S.A. & GUAYAS & 61.671 .551 & 25.440 .826 \\
\hline INMOBILIARIA DEL SOL S.A. MOBILSOL & GUAYAS & 21.017 .730 & 38.247 .118 \\
\hline CODANAS.A. & GUAYAS & 19.453 .943 & 8.703 .936 \\
\hline PROMOTORES INMOBILIARIOS PRONOBIS S.A. & GUAYAS & 15.347 .070 & 15.158 .629 \\
\hline ECOELECTRIC S.A. & GUAYAS & 11.537 .348 & 16.414 .319 \\
\hline BEAUPORT S.A. & GUAYAS & 9.018 .506 & 16.795 .765 \\
\hline BAMBOO EXPORT S.A. BAMPORT & GUAYAS & 5.953 .918 & 4.941.121 \\
\hline GULKANA S.A & GUAYAS & 5.294 .260 & 24.057 .628 \\
\hline CONSORCIO NOBISS.A. & GUAYAS & 3.247 .880 & 149.046 \\
\hline SOLCENTROS.A. & PICHINCHA & 3.057 .495 & 2.909 .557 \\
\hline MEDITECNOS.A. & GUAYAS & 2.122 .426 & 1.210 .288 \\
\hline HACIENDA SAN RAFAEL S.A. H.S.R. & GUAYAS & 1.749 .843 & 7.454 .283 \\
\hline 105 Empresas/Razones Sociales & & 302.420 .204 & 369.252 .305 \\
\hline
\end{tabular}

\section{EVOLUCIÓN DE LOS PRINCIPALES GRUPOS EN 2013-15}

Dado que el número de grupos y la composición de los mismos ha venido cambiando sustancialmente entre el 2007 y el 2015, el siguiente cuadro compara los ingresos de los principales grupos tomando como referencia sus ingresos en 2013 y 2015 (considerando, además, que el país entró en una recesión en el 2015, con el colapso de los precios internacionales del petróleo).
El resultado es el cuadro 15. La verdad es que están en juego demasiados factores: los ingresos de los grupos vinculados a la producción petrolera y la importación tienden a caer; el Grupo Schlumberger no pudo ser incluido, dado que el SRI no lo había identificado en el 2013. Los ingresos del ISSFA sí están reportados en el 2013, pero no en el 2015, por lo cual hay una caída sustanti- 
va en los ingresos de dicho grupo.

En definitiva, los ingresos de estos 12 grupos caen en $2 \%$ entre los dos años, en tanto que el Valor Agregado Bruto total del país aumentó en $5 \%$ en términos nominales. Cabe destacar que el total de ingresos de los grupos económicos aumenta significativamente (26\%), principalmente como efecto del aumento del número de grupos identificados (de 125 a 200). La participación en el PIB de los grupos económicos identificados por el SRI aumenta del $48 \%$ en el 2013 al 57 $\%$ en el 2015, evidenciando un alto nivel de concentración y centralización del capital en el país (mayor al encontrado en los años 1970s por Navarro y en los años 1980s por este autor).

\section{Cuadro 15. Evolución de Ingresos de Principales Grupos, 2013-15}

\begin{tabular}{|l|r|r|r|}
\hline Grupo Económico & Ingresos $\mathbf{2 0 1 3}$ & Ingresos $\mathbf{2 0 1 5}$ & Variación \\
\hline (en millones de dólares corrientes) & \multicolumn{2}{|c|}{} \\
\hline Pichincha / Diners & 1.630 & 1.901 & $17 \%$ \\
\hline OCP / Repsol & 2.388 & 1.835 & $-23 \%$ \\
\hline Eljuri / Austro & 2.176 & 1.868 & $-14 \%$ \\
\hline Wright / Favorita & 2.386 & 2.695 & $13 \%$ \\
\hline Claro / Slim & 1.736 & 1.607 & $-7 \%$ \\
\hline PRONACA & 957 & 1.803 & $88 \%$ \\
\hline Lasso / Banco de Guayaquil & 395 & 440 & $11 \%$ \\
\hline ProduBanco & 307 & 342 & $11 \%$ \\
\hline El Rosado / Czarninski & 1.128 & 1.497 & $33 \%$ \\
\hline Holding DINE / ISSFA 1,224 & 1.224 & 261 & $-79 \%$ \\
\hline Álvaro Noboa & 826 & 549 & $-34 \%$ \\
\hline Isabel Noboa / NOBIS & 274 & 302 & $10 \%$ \\
\hline $\mathbf{1 2}$ Grupos & $\mathbf{1 5 . 4 2 7}$ & $\mathbf{1 5 . 1 0 0}$ & $\mathbf{- 2} \%$ \\
\hline Total GE (125 en 2013, 200 en 2015) & 45.685 & 57.534 & $26 \%$ \\
\hline VAB nominal & $\mathbf{4 8 . 0} \%$ & 100.176 & $5 \%$ \\
\hline GE / VAB & & $\mathbf{5 7 . 4} \%$ & \\
\hline
\end{tabular}


Pástor (2016) cita a varios autores que indican que el gobierno actual ha velado por "el conjunto del capital, aun si eso significa afectar y enfrentarse con algunos sectores particulares" (Unda y Bethania, 2010, p. 22); el capitalismo de Estado sirve a los intereses generales de la clase dominante. Machado (2014) destaca que los grupos económicos se han beneficiado y vinculado privilegiadamente al gobierno de la revolución ciudadana, y el modelo de acumulación no ha cambiado, con una estructura productiva de escaso valor agregado, dependencia petrolera, alto consumo de bienes y servicios importados, economía comercial y de intermediarios, y alta concentración económica (citado por Pástor, pp. 78-79). Ospina, por su parte, indica que en la última década "se ha producido una reducción del poder relativo de los bancos y los agroexportadores a favor de los importadores, de los comerciantes dedicados al mercado interno, así como a favor de las empresas constructoras y proveedoras de otros servicios demandados por el Estado" (Ospina, 2015, p. 6). Los más afectados han sido los agroexportadores como Noboa, UBESA (Standard) y Reybanpac (Wong), mientras que se han consolidado los grupos dedicados al mercado interno (Favorita, El Rosado, Eljuri, TIA, Difare, PRONACA). También se han beneficiado las empresas vinculadas a la construcción (Hidalgo \& Hidalgo, Herdoíza Crespo, HOLCIM, Disensa), y las nuevas empresas petroleras privadas (citado por Pástor, 2016, p. 81).

Pástor destaca que solo cuatro grupos económicos "han desaparecido casi completamente: el grupo Gorelik, el grupo Granda Centeno, el grupo Isaías, y el grupo Umpiérrez" (ibid., p. 83). De estos, el grupo Isaías desplazó sus restantes capitales al exterior, ante la incautación efectuada por el gobierno.

\section{POLÍTICAS ANTI-MONOPÓLICAS Y PRO-COMPETITIVAS}

Un cambio legal importante fue la desvinculación de las instituciones financieras de otro tipo de compañías, al prohibirse que los accionistas de entidades financieras pudiesen a la vez invertir en otros tipos de entidades. No obstante, como se ha visto, se mantienen lazos evidentes entre ciertos grupos económicos y determinadas instituciones financieras (por ejemplo, entre el Banco del Austro y el Grupo Eljuri, como lo confirma el SRI). La desvinculación partiría de la noción de que la coexistencia de entidades financieras y productivas en un solo grupo podría llevar a un conflicto de interés en la entrega y uso del crédito, "lo que 
podría conllevar abusos e incluso, contribuir a la ocurrencia de crisis financieras" (Granda Kuffó, 2011, p. 5).

De igual manera, se prohibió a los accionistas en instituciones financieras la inversión en medios de comunicación, y se reguló la propiedad de los medios (por ejemplo, que sus accionistas tampoco podrían tener acciones en empresas de otros rubros; y que extranjeros no podrían ser accionistas de medios nacionales). No obstante, poco después de expedida la Ley de Comunicación, un conglomerado mexicano compró el Diario "El Comercio" de Quito, a la par que ya era propietario de varios canales de televisión y radio. "El grupo mediático de Ángel González crece en Ecuador. Acumula ya tres canales, once radios y dos diarios. Los controla a través de familiares, abogados o empleados" (El Universo, 22 de febrero, 2015, https://goo.gl/A0Gvq6). El Grupo Eljuri también continuaría siendo propietario de dos canales de televisión, según el SRI.

Se constituyó la Superintendencia de Control de Poder de Mercado, junto a la aprobación de su respectiva Ley, la cual ha impulsado algunas políticas anti-monopólicas y pro-competitivas. Cabe destacar multas a empresas como CLARO, La Fabril y una empresa contratista del IESS (Recapt), y se ha requerido que la etiquetación de ciertos productos se modifique. Se autorizó recientemente la fusión de dos empresas cerveceras que tienen un dominio del $90 \%$ del mercado (Ambev y SAB Miller). A la fecha, no se han desarticulado grupos económicos ni oligopolios importantes en varios sectores. Cabe anotar que en la Constitución de Montecristi se establece, en el Art. 335, que el Estado debe regular, controlar e intervenir, cuando sea necesario, "para evitar cualquier práctica de monopolio u oligopolio privado o de abuso de posición de dominio en el mercado, así como otras prácticas de competencia desleal".

Otro cambio importante, especialmente para la investigación económica, fue que el Servicio de Rentas Internas (SRI) comenzó a compilar y publicar información sobre los grupos económicos. En mi caso, y me imagino que el de otros investigadores en décadas pasadas, se requería una labor de hormiga, recortando balances publicados en la prensa, folletos publicados por los grupos financieros, información sobre miembros de directorios, y hasta direcciones de correo postal, para poder efectuar la investigación; ahora, basta con ir al sitio de Internet del SRI y de la Superintendencia de Bancos para obtener información oficial.

En definitiva, está claro que, al margen de la retórica sobre "socialismo del siglo XXI"y "revolución ciudadana", los principales grupos económicos se han fortalecido en la última década, lo cual destaca la importancia de profundizar aún más la investigación sobre el tema. 


\section{REFERENCIAS BIBLIOGRÁFICAS}

Aguilar, Alonso. Estado, Capitalismo y Clase en el Poder en México. México: Editorial Nuestro Tiempo, 1983.

Brito, Vanessa et al., 2016 Ranking Empresarial, Principales Empresas del País. Quito, Revista Gestión No. 267, septiembre/octubre 2016.

Brito, Vanessa et al., 2016 Ranking. Principales Instituciones Financieras del País. Quito: Revista Gestión No. 264, junio/julio 2016.

Bocarra, Paul. Capitalismo Monopolista de Estado. México: Colección 70, No. 90, Editorial Grijalbo, 1970.

Brownrigg, Leslie Ann. The Agrarian Elite for Southern Ecuador. Nueva York: Tesis Doctoral, Universidad de Columbia. 1972.

Cavagnaro, Jorge (ed.). 500 Mayores Empresas del Ecuador. Impacto de la Caída. Guayaquil: Revista Vistazo No. 1178, 23 de septiembre, 2016. Conaghan, Catherine. Industrialists and the reformist interregnum: dominant class behavior and ideology in Ecuador. Cambridge, EE.UU.: Universidad de Yale. Tesis Doctoral, 1983.

Del Llano, Eduardo (ed.), El Imperialismo, Capitalismo Monopolista. La Habana: Editorial Orbe, 1978.

Diario "El Universo", "Un magnate de Es- tados Unidos controla 10 medios nacionales". Guayaquil: Diario El Universo, 22 de febrero, 2015.

Fierro Carrión, Luis. Los Grupos Financieros en el Ecuador. Quito: Centro de Educación Popular - CEDEP, 1991.

Fierro Carrión, Luis. Los Grupos Monopólicos en el Ecuador: Ensayo de Difusión Popular. Quito: CEDIS, 1986.

Granda Kuffó, María Luisa. "Grupos Económicos en el Ecuador: Supervivencia u Oportunismo", en Revista E+E. Guayaquil: ESPAE, Julio-Septiembre, 2011.

Granja, María. La Burguesía Bancaria, 1980-1984. Quito: Editorial Abya-Yala, 1987. Guerrero, Matilde, La Burguesía Exportadora 19801984. Quito: Editorial Abya-Yala, 1987.

Hanson, David. Political decision making in Ecuador: the influence of business groups.

Gainesville, Florida: Universidad de Florida. Tesis Doctoral, 1971.

Hilferding, Rudolf. El capital Financiero. México: Editorial Tecnos, 1979.

Lenin, Vladimir. El Imperialismo, Fase Superior del Capitalismo. México: Editorial Grijalbo, 1917.

Machado, Decio, Las élites económicas: 
los verdaderos beneficiarios del Gobierno de Rafael Correa. Sitio Rebelión (http://www.rebelion. org/docs/145047.pdf), 2012.

Mancheno, Diego. De la Concentración y

Centralización del Capital al CME: Un Acercamiento al Caso Ecuatoriano. Quito: Tesis de Grado, PUCE, 1986.

Moncada, José. Capitalismo, Burguesía y

Crisis. Quito: IIE-UCE, 1983.

Navarro Jiménez, Guillermo. Los Poderes

Fácticos, II Parte. Quito: Ediciones Zitra, 2006.

Navarro, Guillermo, La Concentración de Capitales en el Ecuador, 1976, Quito: Ediciones Solitierra.

Ospina Peralta, Pablo. Grandes empresas, crisis económica y revolución ciudadana. Quito: Informe de coyuntura CEP, noviembre 2015

Pacheco Prado, Lucas. El Estado y la Acumulación de Capital en el Ecuador. Cuenca: Mimeo, 1979.

Pástor Pazmiño, Carlos, Los Grupos Económicos en el Ecuador, Mimeo, 2015.

Proyecto de Investigación IIE-UCE, Informes del Proyecto Investigación sobre la Concentración y Centralización del Capital en el Ecuador. Quito: Mimeo, IIE-UCE, 1987-89.

Rosero Delgado, Gabriela Alexandra. Oli- garquías en el Ecuador desde el auge neoliberal hasta su ulterior recomposición en la crisis económica del 2000. Quito: Tesis de Maestría, FLACSO, 2013.

SENPLADES: Empresas públicas y planificación: Su rol en la transformación social y productiva. Quito: SENPLADES, 2013

SRI (2016), Conformación de los Grupos Económicos y su Comportamiento Tributario (hoja de Excel). Descargado en septiembre 2016 de: http://www.sri.gob.ec/

Totoy Moreno, David y Esteban Vizuete. Estudio de los Principales Grupos Económicos con Mayores Ingresos en el Ecuador, periodo 2006-2010. Estructura y Comportamiento en el Mercado Nacional. Quito: Tesis de Grado, UCE. 2012.

Unda, Mario, y Ellis Bethania. "Burguesía 'ciudadana', nueva hegemonía, nueva alianza de clases". Revista "R", 2010: 19-31.

Villalta Ordóñez, Walter Fernando. Contribución a la recaudación por Impuesto a la Renta de los grupos económicos más representativos de Ecuador, 2010-2012. Guayaquil: Tesis de Magister en Tributación y Finanzas, Universidad de Guayaquil, 2014. 